\title{
Distributed fusion estimation from measurements with correlated random parameter matrices and noise correlation
}

\author{
Raquel Caballero-Águila, Irene García-Garrido \& Josefa Linares-Pérez
}

\section{Preprint version. Please cite original version:}

Raquel Caballero-Águila, Irene García-Garrido \& Josefa Linares-Pérez (2020). Distributed fusion estimation from measurements with correlated random parameter matrices and noise correlation, International Journal of Computer Mathematics, 97 (1-2), 95-108.

https://doi.org/10.1080/00207160.2018.1437264

\section{Abstract}

This paper addresses the distributed fusion estimation problem for discrete-time multi-sensor stochastic systems with random parameter matrices. It is assumed that the random parameter matrices in the observation equations are one-step autocorrelated and cross-correlated between the different sensors and the additive noises are also correlated. Under these assumptions, a recursive algorithm is proposed to obtain local least squares linear filters based on the measurements of each sensor, and the distributed fusion filter is designed as the matrix-weighted linear combination of these estimators which minimizes the mean squared estimation error. This research is illustrated by two numerical simulation examples where multi-sensor systems with randomly delayed measurements and missing measurements are considered, respectively, and the performance of the proposed estimators is analysed by comparing the estimation error variances of the distributed and centralized fusion filters. 


\section{Introduction}

In recent years, the use of sensor networks has received significant attention in many practical domains, since they usually provide more information than traditional singlesensor communication systems. For this reason, the fusion estimation problem in sensor network stochastic systems has been widely studied in many fields of science, technology and military such as target tracking, navigation and detection (see [11] among others).

Although there are several information fusion techniques, the most common fusion estimation approaches are the centralized and distributed ones. The former is based on the measurements from all the sensors, which are sent to a fusion centre, and so, it provides the optimal estimator when all the sensors work accurately. However, a sensor error can spoil the performance of the centralized filter and may give rise to heavy computational burden and poor reliability. In the distributed fusion estimation approach, each single sensor sends a local estimator to the fusion centre, where the state is estimated by a combination of all the received local filters using a certain optimality criterion. Thus, the distributed approach has lower estimation accuracy, which is compensated with considerable advantages, such as greater robustness, reliability and flexibility due to its parallel structure. Therefore, the distributed fusion method is usually more attractive and has become an interesting research topic (see e.g. [1-5], $[14-16],[19,20]$, and references therein). In [17], a survey on distributed fusion estimation algorithms for multi-sensor networked systems based on the existing literature is presented and new advances are discussed.

In general, there are many situations with network-induced phenomena, such as multiplicative noise uncertainties, random delays, packet dropouts and missing measurements, in which the state estimation problem can be addressed by transforming the original system into one with random parameter matrices. For example in [14] and [21] systems with packet dropouts and/or random delays are transformed into systems with random parameter matrices. Systems with multiplicative noises in the state and observation equations as those investigated in [19] are special cases of this kind of systems. Also, in complex stochastic systems it is extremely important to have the missing measurement phenomenon in mind for the derivation of the estimation algorithms, and many observation models for time-varying complex networks with missing measurements, as those considered in [9] or those for nonlinear time-varying systems subject to multiplicative noises and missing measurements in [10], can be defined by using random measurement matrices. The optimal filtering problem is addressed for a class of discrete-time stochastic systems with multiplicative noises and random sensor delays in [6] and, later, also with missing measurements in [7], by transforming the original system into one with random parameter matrices.

Discrete-time systems with random parameter matrices arise in many areas, such as radar control, missile track estimation, satellite navigation, digital control of chemical processes or economic systems, among others [13]. Accordingly, the study of the estimation problem in this kind of systems has become an active research field. In [13] a distributed Kalman filtering fusion is proposed for systems with independent random state transition and measurement matrices and white noises, which is also applied to multi-model dynamic processes. For a class of discrete-time multi-sensor stochastic systems also with independent random parameter matrices but autocorrelated and cross-correlated noises, the centralized fusion estimation problem has been addressed under the phenomena of fading measurements in [8]. Moreover, by considering this same correlation assumption of the noise processes and one-step correlated 
and cross-correlated random measurement matrices, the centralized fusion linear filter is obtained in [12], where the results are applied to systems with missing measurements and randomly delayed observations. More recently, considering correlated random parameter matrices at the same sampling time, in [18] centralized fusion estimators are presented for a class of nonlinear discrete-time stochastic systems with fading measurements and multi-step correlated noises. The distributed fusion estimation problem has also been studied for sensor network systems with independent random parameter matrices and correlated noises in [1] and also in [4] where, additionally, random packet dropouts are assumed to occur during transmission. Centralized and distributed fusion estimation problems are both studied in $[2,3,5]$ for networked systems with random parameter matrices and different correlation assumptions about the noises involved in the system. Specifically, measurements subject to random delays during the transmission are considered in [2] and measurements subject to both random delays and packet dropouts are analyzed in $[3,5]$.

Motivated by the above discussion, this paper deals with the distributed fusion estimation problem for discrete-time linear stochastic systems with multi-sensor measurements including correlated and cross-correlated random parameter matrices and noises. To the best of the authors' knowledge, the distributed fusion estimation problem in this kind of complex stochastic systems has not yet been investigated, so it is an interesting research topic. The main contributions of this paper can be highlighted as follows: (a) The considered systems provide a unified framework for simultaneously dealing with some network-induced phenomena, such as correlated randomly delayed measurements and missing measurements, or uncertainties caused by multiplicative noises, along with correlated and cross-correlated noises; hence, the proposed distributed fusion filter has wide applicability. (b) Unlike [12], where centralized fusion estimators are obtained, this paper proposes a distributed fusion estimator, which has greater robustness and fault-tolerance abilities in comparison with the centralized fusion estimator and its computational methodology is simple. (c) The innovation technique is used to obtain algorithms for the local least-squares linear filtering estimators and the proposed distributed fusion filter is designed as a matrix-weighted sum of the local filters with the aim of minimizing the mean squared estimation error. (d) The proposed observation model is more general than the one considered in [1], where the distributed fusion filtering estimators are obtained for networked systems with independent random state transition and measurement matrices, whereas correlation of the random parameter matrices in the observation equation is considered in the current paper.

The rest of the paper is organized as follows. Section 2 describes the system model with correlated and cross-correlated random parameter matrices and noises, specifying the assumptions under which the distributed fusion estimation problem is addressed. In Section 3, the local least squares linear filtering algorithm is given, and the proposed distributed fusion filter is designed by a matrix-weighted linear combination of the local filters using the mean squared error optimality criterion. Two illustrative simulation examples are given in Section 4 to show the applicability of the proposed filtering algorithm. Finally, some conclusions are drawn in Section 5. 


\section{System model}

Let us consider the following discrete-time linear stochastic system with measurements coming from $m$ different sensors:

$$
\begin{aligned}
& x_{k+1}=F_{k} x_{k}+w_{k}, \quad k \geq 0, \\
& y_{k}^{(i)}=H_{k}^{(i)} x_{k}+B_{k}^{(i)} v_{k}^{(i)}, \quad k \geq 1, \quad i=1, \ldots, m,
\end{aligned}
$$

where $x_{k} \in \mathbb{R}^{n_{x}}$ is the state vector to be estimated and $y_{k}^{(i)} \in \mathbb{R}^{n_{y}}, i=1, \ldots, m$, is the output measurement of the $i$-th sensor at the sampling time $k . w_{k} \in \mathbb{R}^{n_{x}}$ and $v_{k}^{(i)} \in \mathbb{R}^{n_{v}}$ are the process and measurement noise vectors, respectively. $F_{k}$ is the state transition matrix and $H_{k}^{(i)}$ and $B_{k}^{(i)}$ are the measurement matrices, all of them with random parameters and suitable dimensions.

The following assumptions about the initial state, the random parameter matrices and the noises involved in system (1) are required.

Assumption 1. The initial state $x_{0}$ is a zero-mean random vector with $\operatorname{Cov}\left[x_{0}\right]=\Sigma_{0}$. Also, it is assumed to be independent of the random parameter matrices and noise processes.

Assumption 2. The random parameter matrices $\left\{F_{k} ; k \geq 0\right\},\left\{H_{k}^{(i)} ; k \geq 1\right\}$ and $\left\{B_{k}^{(i)} ; k \geq 1\right\}$ have known means, which will be denoted $\bar{F}_{k} \equiv E\left[F_{k}\right], \bar{H}_{k}^{(i)} \equiv E\left[H_{k}^{(i)}\right]$ and $\bar{B}_{k}^{(i)} \equiv E\left[B_{k}^{(i)}\right], i=1, \ldots, m$. Also, for $i, j=1, \ldots, m$, the following expectations are assumed to be known

$$
\begin{aligned}
E\left[f_{p q}(k) f_{p^{\prime} q^{\prime}}(s)\right]= & E\left[f_{p q}(k) f_{p^{\prime} q^{\prime}}(k)\right] \delta_{k, s}, \\
E\left[h_{p q}^{(i)}(k) h_{p^{\prime} q^{\prime}}^{(j)}(s)\right]= & E\left[h_{p q}^{(i)}(k) h_{p^{\prime} q^{\prime}}^{(j)}(k)\right] \delta_{k, s}+E\left[h_{p q}^{(i)}(k) h_{p^{\prime} q^{\prime}}^{(j)}(k-1)\right] \delta_{k-1, s}, \quad s \leq k, \\
E\left[b_{p q}^{(i)}(k) b_{p^{\prime} q^{\prime}}^{(j)}(s)\right]= & E\left[b_{p q}^{(i)}(k) b_{p^{\prime} q^{\prime}}^{(j)}(k)\right] \delta_{k, s}+E\left[b_{p q}^{(i)}(k) b_{p^{\prime} q^{\prime}}^{(j)}(k-1)\right] \delta_{k-1, s}, \quad s \leq k, \\
E\left[h_{p q}^{(i)}(k) b_{p^{\prime} q^{\prime}}^{(j)}(s)\right]= & E\left[h_{p q}^{(i)}(k) b_{p^{\prime} q^{\prime}}^{(j)}(k)\right] \delta_{k, s}+E\left[h_{p q}^{(i)}(k) b_{p^{\prime} q^{\prime}}^{(j)}(k-1)\right] \delta_{k-1, s} \\
& +E\left[h_{p q}^{(i)}(k) b_{p^{\prime} q^{\prime}}^{(j)}(k+1)\right] \delta_{k+1, s},
\end{aligned}
$$

where $f_{p q}(k), h_{p q}^{(i)}(k)$ and $b_{p q}^{(i)}(k)$ denote the $(p, q)$-th entries of matrices $F_{k}, H_{k}^{(i)}$ and $B_{k}^{(i)}$, respectively.

Assumption 3. $\left\{w_{k} ; k \geq 0\right\}$ and $\left\{v_{k}^{(i)} ; k \geq 1\right\}, i=1, \ldots, m$, are zero-mean sequences and the following covariances and cross-covariances are known

$$
\begin{aligned}
& E\left[w_{k} w_{s}^{T}\right]=Q_{k} \delta_{k, s}+Q_{k, k-1} \delta_{k-1, s}, \quad s \leq k, \\
& E\left[v_{k}^{(i)} v_{s}^{(j) T}\right]=R_{k}^{(i j)} \delta_{k, s}+R_{k, k-1}^{(i j)} \delta_{k-1, s}, \quad s \leq k, \quad i, j=1, \ldots, m, \\
& E\left[w_{k} v_{s}^{(i) T}\right]=S_{k}^{(i)} \delta_{k, s}+S_{k, k+1}^{(i)} \delta_{k+1, s}+S_{k, k+2}^{(i)} \delta_{k+2, s}, \quad i=1, \ldots, m .
\end{aligned}
$$

Assumption 4. Independence assumptions:

- $\left\{F_{k} ; k \geq 0\right\}$ is independent of $\left(\left\{H_{k}^{(i)} ; k \geq 1\right\},\left\{B_{k}^{(i)} ; k \geq 1\right\},\left\{w_{k} ; k \geq 0\right\},\left\{v_{k}^{(i)} ; k \geq 1\right\}, i=1, \ldots, m\right)$. 


$$
\begin{aligned}
- & \left(\left\{H_{k}^{(i)} ; k \geq 1\right\},\left\{B_{k}^{(i)} ; k \geq 1\right\}, i=1, \ldots, m\right) \text { is independent of } \\
& \left(\left\{w_{k} ; k \geq 0\right\},\left\{v_{k}^{(i)} ; k \geq 1\right\}, i=1, \ldots, m\right) .
\end{aligned}
$$

The basic model in which $\left\{H_{k}^{(i)} ; k \geq 1\right\}$ and $\left\{B_{k}^{(i)} ; k \geq 1\right\}, i=1, \ldots, m$, are independent sequences of independent random parameter matrices can be unrealistic in many practical situations, and the fusion estimation algorithms must be modified to incorporate the effect of different types of correlation between the matrices. The correlation at consecutive sampling times considered in Assumption 2 is appropriate, for example, to model real situations involving missing or delayed measurements in physical plants provided with stand-by sensors for the immediate replacement of a failed unit, thus avoiding the possibility of successive observation failures. This type of correlation among consecutive missing or delayed packets usually arises when the packets are transmitted in a data stream through an intermediate node. For example, in systems with random transmission delays, if more packets arrive before the first packet leaves, the delays of new arrivals will depend on the current packet processing delay. In the study of packet transmission delays in a data stream for different transmission rates and different packet sizes, significant correlations among delay values of successive packets are found at high sending rates.

Assumption 3 on the correlation between the sensor noises $\left\{v_{k}^{(i)} ; k \geq 1\right\}, i=$ $1, \ldots, m$, and the process noise $\left\{w_{k} ; k \geq 0\right\}$ has been usually considered in the literature, since the independence of the noises is not realistic in many practical situations (for example, when all the sensors operate in the same noisy environment or the sensor measurement noises depend on the signal), so noise correlation provides a more comprehensive framework to model some real-world complexities. Actually, even more general correlation structures than the one established in Assumption 3 could be considered. However, this is the most common assumption used in papers involving correlated noises, since the approach and methodology under more general correlation assumptions would be similar and just additional complexity in the mathematical derivations would be implied.

The following property will be used to calculate some expectations involving the random parameter matrices $F_{k}, H_{k}^{(i)}$ and $B_{k}^{(i)}, i=1, \ldots, m$ :

$$
\text { Let } A=\left(a_{r s}\right)_{\substack{r=1, \ldots, n_{1} \\ s=1, \ldots, n_{2}}}, B=\left(b_{r s}\right)_{\substack{r=1, \ldots, m_{1} \\ s=1, \ldots, m_{2}}} \text { and } C=\left(c_{r s}\right)_{\substack{r=1, \ldots, n_{2} \\ s=1, \ldots, m_{2}}} \text { be random }
$$
parameter matrices, such that $C$ is independent of $(A, B)$; then, the $(p, q)$-th entries of the matrix $E\left[A C B^{T}\right]$ are given by

$$
\left(E\left[A C B^{T}\right]\right)_{p q}=\sum_{r=1}^{n_{2}} \sum_{s=1}^{m_{2}} E\left[a_{p r} b_{q s}\right] E\left[c_{r s}\right], \quad p=1, \ldots, n_{1}, \quad q=1, \ldots, m_{1} .
$$

Our aim is to address the least squares (LS) linear filtering problem in this class of systems with correlated and cross-correlated random parameter matrices and noises using the distributed fusion approach. 


\section{Distributed fusion filtering estimators}

In this section, the distributed fusion filter of the state $x_{k}$ based on the available measurements $y_{1}^{(i)}, \ldots, y_{k}^{(i)}, i=1, \ldots, m$, is designed. First, at each sensor, a local LS linear filtering recursive algorithm is obtained using its own measurement data. Then, these estimators are sent to the fusion centre where the distributed fusion filter is computed.

\subsection{Local LS linear filters}

In order to simplify the expressions of the local linear filtering estimators and the subsequent calculations, let us first present the following properties [12]:

- The matrices $\mathcal{D}_{k} \equiv E\left[x_{k} x_{k}^{T}\right]$ and $\mathcal{D}_{k, k-1} \equiv E\left[x_{k} x_{k-1}^{T}\right]$ are recursively calculated by

$$
\begin{aligned}
& \mathcal{D}_{k}=E\left[F_{k-1} \mathcal{D}_{k-1} F_{k-1}^{T}\right]+\bar{F}_{k-1} Q_{k-2, k-1}+Q_{k-1, k-2} \bar{F}_{k-1}^{T}+Q_{k-1}, \quad k \geq 2 ; \\
& \mathcal{D}_{1}=E\left[F_{0} \mathcal{D}_{0} F_{0}^{T}\right]+Q_{0} ; \quad \mathcal{D}_{0}=\Sigma_{0}, \\
& \mathcal{D}_{k, k-1}=\bar{F}_{k-1} \mathcal{D}_{k-1}+Q_{k-1, k-2}, \quad k \geq 2 ; \quad \mathcal{D}_{1,0}=\bar{F}_{0} \mathcal{D}_{0} .
\end{aligned}
$$

- The noise processes $\left\{w_{k} ; k \geq 0\right\}$ and $\left\{v_{k}^{(i)} ; k \geq 1\right\}, i=1, \ldots, m$, satisfy the following correlation properties

$$
\begin{aligned}
& \mathcal{W}_{k}^{(i)} \equiv E\left[w_{k} y_{k}^{(i) T}\right]=Q_{k, k-1} \bar{H}_{k}^{(i) T}+S_{k}^{(i)} \bar{B}_{k}^{(i) T}, \quad k \geq 1, \\
& \mathcal{E}_{k}^{(i)} \equiv E\left[x_{k} v_{k}^{(i) T}\right]=\bar{F}_{k-1} S_{k-2, k}^{(i)}+S_{k-1, k}^{(i)}, \quad k \geq 2 ; \quad \mathcal{E}_{1}^{(i)}=S_{0,1}^{(i)}, \\
& \mathcal{E}_{k, k-1}^{(i)} \equiv E\left[x_{k} v_{k-1}^{(i) T}\right]=\bar{F}_{k-1} \mathcal{E}_{k-1}^{(i)}+S_{k-1}^{(i)}, \quad k \geq 2, \\
& \mathcal{V}_{k, k-1}^{(i j)} \equiv E\left[B_{k}^{(i)} v_{k}^{(i)} y_{k-1}^{(j) T}\right]=E\left[B_{k}^{(i)} S_{k-2, k}^{(i) T} H_{k-1}^{(j) T}\right]+E\left[B_{k}^{(i)} R_{k, k-1}^{(i j)} B_{k-1}^{(j) T}\right], \quad k \geq 2 .
\end{aligned}
$$

For each sensor $i=1, \ldots, m$, a recursive algorithm for the local LS linear filter, $\widehat{x}_{k / k}^{(i)}$, together with its filtering error covariance matrix, $\Sigma_{k / k}^{(i)}$, is given in the following theorem.

Theorem 3.1. For system (1), under assumptions 1-4, the local LS linear filter for the $i$-th sensor, $\widehat{x}_{k / k}^{(i)}, i=1, \ldots, m$, is given by

$$
\widehat{x}_{k / k}^{(i)}=\widehat{x}_{k / k-1}^{(i)}+\mathcal{X}_{k}^{(i)} \Pi_{k}^{(i)-1} \mu_{k}^{(i)}, \quad k \geq 1 ; \quad \widehat{x}_{0 / 0}^{(i)}=0,
$$

where the one-step predictor, $\widehat{x}_{k / k-1}^{(i)}$, is calculated by

$$
\widehat{x}_{k / k-1}^{(i)}=\bar{F}_{k-1} \widehat{x}_{k-1 / k-1}^{(i)}+\mathcal{W}_{k-1}^{(i)} \Pi_{k-1}^{(i)-1} \mu_{k-1}^{(i)}, \quad k \geq 2 ; \quad \widehat{x}_{1 / 0}^{(i)}=0 .
$$

The innovation, $\mu_{k}^{(i)}$, is obtained by

$$
\mu_{k}^{(i)}=y_{k}^{(i)}-\bar{H}_{k}^{(i)} \widehat{x}_{k / k-1}^{(i)}-\mathcal{Y}_{k, k-1}^{(i)} \Pi_{k-1}^{(i)-1} \mu_{k-1}^{(i)}, \quad k \geq 2 ; \quad \mu_{1}^{(i)}=y_{1}^{(i)},
$$


where, denoting $\widetilde{H}_{k}^{(i)} \equiv H_{k}^{(i)}-\bar{H}_{k}^{(i)}$, the matrix $\mathcal{Y}_{k, k-1}^{(i)} \equiv E\left[y_{k}^{(i)} \mu_{k-1}^{(i) T}\right]$ satisfies

$$
\mathcal{Y}_{k, k-1}^{(i)}=E\left[\widetilde{H}_{k}^{(i)} \mathcal{D}_{k, k-1} H_{k-1}^{(i) T}\right]+E\left[\widetilde{H}_{k}^{(i)} \mathcal{E}_{k, k-1}^{(i)} B_{k-1}^{(i) T}\right]+\mathcal{V}_{k, k-1}^{(i)}, \quad k \geq 2 .
$$

The matrix $\mathcal{X}_{k}^{(i)} \equiv E\left[x_{k} \mu_{k}^{(i) T}\right]$ is obtained by

$$
\begin{aligned}
\mathcal{X}_{k}^{(i)} & =\Sigma_{k / k-1}^{(i)} \bar{H}_{k}^{(i) T}+\mathcal{E}_{k}^{(i)} \bar{B}_{k}^{(i) T}-\mathcal{X}_{k, k-1}^{(i)} \Pi_{k-1}^{(i)-1} \mathcal{Y}_{k, k-1}^{(i) T}, \quad k \geq 2 \\
\mathcal{X}_{1}^{(i)} & =\Sigma_{1 / 0}^{(i)} \bar{H}_{1}^{(i) T}+\mathcal{E}_{1}^{(i)} \bar{B}_{1}^{(i) T}
\end{aligned}
$$

where $\mathcal{X}_{k, k-1}^{(i)} \equiv E\left[x_{k} \mu_{k-1}^{(i) T}\right]$ is given by $\mathcal{X}_{k, k-1}^{(i)}=\bar{F}_{k-1} \mathcal{X}_{k-1}^{(i)}+\mathcal{W}_{k-1}^{(i)}, k \geq 2$.

The innovation covariance matrix, $\Pi_{k}^{(i)}$, satisfies

$$
\begin{aligned}
\Pi_{k}^{(i)}= & E\left[\widetilde{H}_{k}^{(i)} \mathcal{D}_{k} H_{k}^{(i) T}\right]+E\left[\widetilde{H}_{k}^{(i)} \mathcal{E}_{k}^{(i)} B_{k}^{(i) T}\right]+E\left[B_{k}^{(i)} \mathcal{E}_{k}^{(i) T} \widetilde{H}_{k}^{(i) T}\right]+E\left[B_{k}^{(i)} R_{k}^{(i)} B_{k}^{(i) T}\right] \\
& +\bar{H}_{k}^{(i)} \mathcal{X}_{k}^{(i)}+\mathcal{X}_{k}^{(i) T} \bar{H}_{k}^{(i) T}-\bar{H}_{k}^{(i)} \Sigma_{k / k-1}^{(i)} \bar{H}_{k}^{(i) T}-\mathcal{Y}_{k, k-1}^{(i)} \Pi_{k-1}^{(i)-1} \mathcal{Y}_{k, k-1}^{(i) T}, \quad k \geq 2 ; \\
\Pi_{1}^{(i)}= & E\left[\widetilde{H}_{1}^{(i)} \mathcal{D}_{1} H_{1}^{(i) T}\right]+E\left[H_{1}^{(i)} \mathcal{E}_{1}^{(i)} B_{1}^{(i) T}\right]+E\left[B_{1}^{(i)} \mathcal{E}_{1}^{(i) T} H_{1}^{(i) T}\right] \\
& +E\left[B_{1}^{(i)} R_{1}^{(i)} B_{1}^{(i) T}\right]+\bar{H}_{1}^{(i)} \Sigma_{1 / 0}^{(i)} \bar{H}_{1}^{(i) T}
\end{aligned}
$$

The filtering error covariance matrix, $\Sigma_{k / k}^{(i)}$, is computed by

$$
\Sigma_{k / k}^{(i)}=\Sigma_{k / k-1}^{(i)}-\mathcal{X}_{k}^{(i)} \Pi_{k}^{(i)-1} \mathcal{X}_{k}^{(i) T}, \quad k \geq 1 ; \quad \Sigma_{0 / 0}^{(i)}=\Sigma_{0}
$$

where the prediction error covariance matrix, $\Sigma_{k / k-1}^{(i)}$, is given by

$$
\begin{aligned}
\Sigma_{k / k-1}^{(i)}= & \mathcal{D}_{k}+\bar{F}_{k-1}\left(\Sigma_{k-1 / k-1}^{(i)}-\mathcal{D}_{k-1}\right) \bar{F}_{k-1}^{T}+\mathcal{W}_{k-1}^{(i)} \Pi_{k-1}^{(i)-1} \mathcal{W}_{k-1}^{(i) T} \\
& -\mathcal{X}_{k, k-1}^{(i)} \Pi_{k-1}^{(i)-1} \mathcal{W}_{k-1}^{(i) T}-\mathcal{W}_{k-1}^{(i)} \Pi_{k-1}^{(i)-1} \mathcal{X}_{k, k-1}^{(i) T}, \quad k \geq 2 ; \\
\Sigma_{1 / 0}^{(i)}= & \mathcal{D}_{1} .
\end{aligned}
$$

Finally, the matrices $\mathcal{D}_{k}, \mathcal{D}_{k, k-1}$ and $\mathcal{W}_{k}^{(i)}, \mathcal{E}_{k}^{(i)}, \mathcal{E}_{k, k-1}^{(i)}, \mathcal{V}_{k, k-1}^{(i)}$ are given in (2) and (3), respectively.

Proof. The proof, based on an innovation approach, is analogous to that in [12]. Hence the details are omitted here.

\subsection{Distributed fusion filter weighted by matrices}

As we have already indicated, our aim is to design a distributed fusion filter, $\widehat{x}_{k / k}^{(D)}$, as a matrix-weighted sum of the local filters, $\widehat{x}_{k / k}^{(i)}, i=1, \ldots, m$, such that the optimal weighting matrices are computed to minimize the mean squared estimation error. The following two lemmas provide the expectations required to obtain the proposed fusion estimator. Hereafter, the assumptions and notation used are the same as those in Theorem 3.1. 
Lemma 3.2. For $i, j=1, \ldots, m$ and $i \neq j$, the expectation $L_{k}^{(i j)} \equiv E\left[\widehat{x}_{k / k-1}^{(i)} \mu_{k}^{(j) T}\right]$ satisfies

$$
\begin{aligned}
L_{k}^{(i j)}= & \left(\bar{F}_{k-1} K_{k-1 / k-2}^{(i)} \bar{F}_{k-1}^{T}-K_{k / k-1}^{(i j)}\right) \bar{H}_{k}^{(j) T} \\
& +\mathcal{X}_{k, k-1}^{(i)} \Pi_{k-1}^{(i)-1}\left(\Delta_{k, k-1}^{(j i) T}+\mathcal{V}_{k, k-1}^{(j i) T}\right)-L_{k, k-1}^{(i j)} \Pi_{k-1}^{(j)-1} \mathcal{Y}_{k, k-1}^{(j) T}, \quad k \geq 2 ; \\
L_{1}^{(i j)}= & 0,
\end{aligned}
$$

where the expectation $\Delta_{k, k-1}^{(i j)} \equiv E\left[H_{k}^{(i)} x_{k} \mu_{k-1}^{(j) T}\right]$ is obtained by

$$
\begin{aligned}
& \Delta_{k, k-1}^{(i j)}= E\left[H_{k}^{(i)} \mathcal{D}_{k, k-1} H_{k-1}^{(j) T}\right]+E\left[H_{k}^{(i)} \mathcal{E}_{k, k-1}^{(j)} B_{k-1}^{(j) T}\right] \\
& \quad-\bar{H}_{k}^{(i)} \bar{F}_{k-1}\left(K_{k-1 / k-2}^{(j)} \bar{H}_{k-1}^{(j) T}+\mathcal{X}_{k-1, k-2}^{(j)} \Pi_{k-2}^{(j)-1} \mathcal{Y}_{k-1, k-2}^{(j) T}\right), \quad k \geq 3 \\
& \Delta_{2,1}^{(i j)}=E\left[H_{2}^{(i)} \mathcal{D}_{2,1} H_{1}^{(j) T}\right]+E\left[H_{2}^{(i)} \mathcal{E}_{2,1}^{(j)} B_{1}^{(j) T}\right]-\bar{H}_{2}^{(i)} \bar{F}_{1} K_{1 / 0}^{(j)} \bar{H}_{1}^{(j) T}
\end{aligned}
$$

and $L_{k, k-1}^{(i j)} \equiv E\left[\widehat{x}_{k / k-1}^{(i)} \mu_{k-1}^{(j) T}\right]$ is computed by

$$
L_{k, k-1}^{(i j)}=\bar{F}_{k-1} L_{k-1}^{(i j)}+\mathcal{X}_{k, k-1}^{(i)} \Pi_{k-1}^{(i)-1} \Pi_{k-1}^{(i j)}, \quad k \geq 2 .
$$

The cross-covariance matrices, $K_{k / k-1}^{(i j)} \equiv E\left[\widehat{x}_{k / k-1}^{(i)} \widehat{x}_{k / k-1}^{(j) T}\right]$, between any two local predictors are computed by

$$
\begin{aligned}
K_{k / k-1}^{(i j)}= & \bar{F}_{k-1} K_{k-1 / k-2}^{(i j)} \bar{F}_{k-1}^{T}+\bar{F}_{k-1} L_{k-1}^{(i j)} \Pi_{k-1}^{(j)-1} \mathcal{X}_{k, k-1}^{(j) T} \\
& +\mathcal{X}_{k, k-1}^{(i)} \Pi_{k-1}^{(i)-1} L_{k-1}^{(j i) T} \bar{F}_{k-1}^{T}+\mathcal{X}_{k, k-1}^{(i)} \Pi_{k-1}^{(i)-1} \Pi_{k-1}^{(i j)} \Pi_{k-1}^{(j)-1} \mathcal{X}_{k, k-1}^{(j) T}, \quad k \geq 2, \quad i \neq j ; \\
K_{1 / 0}^{(i j)}=0, \quad i \neq j, & 0 \\
K_{k / k-1}^{(i)}= & \mathcal{D}_{k}-\Sigma_{k / k-1}^{(i)}, \quad k \geq 1 .
\end{aligned}
$$

Proof. We will repeatedly use the equations and assumptions on the system model (1), as well as expression (6) for the innovation.

In this way, the following expression for $L_{k}^{(i j)} \equiv E\left[\widehat{x}_{k / k-1}^{(i)} \mu_{k}^{(j) T}\right]$ is obtained:

$$
L_{k}^{(i j)}=E\left[\widehat{x}_{k / k-1}^{(i)} x_{k}^{T} H_{k}^{(j) T}\right]+E\left[\widehat{x}_{k / k-1}^{(i)} v_{k}^{(j) T} B_{k}^{(j) T}\right]-K_{k / k-1}^{(i j)} \bar{H}_{k}^{(j) T}-L_{k, k-1}^{(i j)} \Pi_{k-1}^{(j)-1} \mathcal{Y}_{k, k-1}^{(j) T},
$$

so we need to calculate the expectations $E\left[\widehat{x}_{k / k-1}^{(i)} x_{k}^{T} H_{k}^{(j) T}\right]$ and $E\left[\widehat{x}_{k / k-1}^{(i)} v_{k}^{(j) T} B_{k}^{(j) T}\right]$.

For the first one, using (5) for $\widehat{x}_{k / k-1}^{(i)}$ with (4) for $\widehat{x}_{k-1 / k-1}^{(i)}$, it is deduced that

$$
\widehat{x}_{k / k-1}^{(i)}=\bar{F}_{k-1} \widehat{x}_{k-1 / k-2}^{(i)}+\mathcal{X}_{k, k-1}^{(i)} \Pi_{k-1}^{(i)-1} \mu_{k-1}^{(i)}, \quad k \geq 2 ; \quad \widehat{x}_{1 / 0}^{(i)}=0 .
$$

According to the Orthogonal Projection Lemma (OPL), since $E\left[\widehat{x}_{k-1 / k-2}^{(i)} x_{k-1}^{T}\right]=$ 
$E\left[\widehat{x}_{k-1 / k-2}^{(i)} \widehat{x}_{k-1 / k-2}^{(i) T}\right]$, from (11) we obtain

$$
E\left[\widehat{x}_{k / k-1}^{(i)} x_{k}^{T} H_{k}^{(j) T}\right]=\bar{F}_{k-1} K_{k-1 / k-2}^{(i)} \bar{F}_{k-1}^{T} \bar{H}_{k}^{(j) T}+\mathcal{X}_{k, k-1}^{(i)} \Pi_{k-1}^{(i)-1} \Delta_{k, k-1}^{(j i) T} .
$$

For the second expectation, using again (11), it is clear that

$$
E\left[\widehat{x}_{k / k-1}^{(i)} v_{k}^{(j) T} B_{k}^{(j) T}\right]=\bar{F}_{k-1} E\left[\widehat{x}_{k-1 / k-2}^{(i)} v_{k}^{(j) T} B_{k}^{(j) T}\right]+\mathcal{X}_{k, k-1}^{(i)} \Pi_{k-1}^{(i)-1} E\left[\mu_{k-1}^{(i)} v_{k}^{(j) T} B_{k}^{(j) T}\right]
$$

and, taking into account that $B_{k}^{(j)} v_{k}^{(j)}$ is independent of $y_{s}^{(i)}$, for $s \leq k-2$, and consequently it is independent of $\mu_{s}^{(i)}$, for $s \leq k-2$, we have that

$$
E\left[\widehat{x}_{k-1 / k-2}^{(i)} v_{k}^{(j) T} B_{k}^{(j) T}\right]=0 \text { and } E\left[\mu_{k-1}^{(i)} v_{k}^{(j) T} B_{k}^{(j) T}\right]=E\left[y_{k-1}^{(i)} v_{k}^{(j) T} B_{k}^{(j) T}\right] .
$$

Then, it is deduced that

$$
E\left[\widehat{x}_{k / k-1}^{(i)} v_{k}^{(j) T} B_{k}^{(j) T}\right]=\mathcal{X}_{k, k-1}^{(i)} \Pi_{k-1}^{(i)-1} \mathcal{V}_{k, k-1}^{(j i) T} .
$$

From the above relations, expression (7) for $L_{k}^{(i j)}$ is immediately derived.

Next, we calculate $(8)$ for $\Delta_{k, k-1}^{(i j)}=E\left[H_{k}^{(i)} x_{k} \mu_{k-1}^{(j) T}\right]=E\left[H_{k}^{(i)} x_{k} y_{k-1}^{(j) T}\right]-$ $E\left[H_{k}^{(i)} x_{k} \widehat{x}_{k-1 / k-2}^{(j) T}\right] \bar{H}_{k-1}^{(j) T}-E\left[H_{k}^{(i)} x_{k} \mu_{k-2}^{(j) T}\right] \Pi_{k-2}^{(j)-1} \mathcal{Y}_{k-1, k-2}^{(j) T}$. By using the conditional expectation properties, we have

$$
\begin{aligned}
\Delta_{k, k-1}^{(i j)}= & E\left[H_{k}^{(i)} \mathcal{D}_{k, k-1} H_{k-1}^{(j) T}\right]+E\left[H_{k}^{(i)} \mathcal{E}_{k, k-1}^{(j)} B_{k-1}^{(j) T}\right] \\
& -E\left[H_{k}^{(i)} x_{k} \widehat{x}_{k-1 / k-2}^{(j) T}\right] \bar{H}_{k-1}^{(j) T}-E\left[H_{k}^{(i)} x_{k} \mu_{k-2}^{(j) T}\right] \Pi_{k-2}^{(j)-1} \mathcal{Y}_{k-1, k-2}^{(j) T} .
\end{aligned}
$$

From the OPL, it is deduced that $E\left[H_{k}^{(i)} x_{k} \widehat{x}_{k-1 / k-2}^{(j) T}\right]=\bar{H}_{k}^{(i)} \bar{F}_{k-1} K_{k-1 / k-2}^{(j)}$, $E\left[H_{k}^{(i)} x_{k} \mu_{k-2}^{(j) T}\right]=\bar{H}_{k}^{(i)} \bar{F}_{k-1} \mathcal{X}_{k-1, k-2}^{(j)}$ and (8) for $\Delta_{k, k-1}^{(i j)}$ is proved.

Finally, expression (9) for $L_{k, k-1}^{(i j)}$ is immediately deduced using (11) for $\widehat{x}_{k / k-1}^{(i)}$ and the derivation of (10) for $K_{k / k-1}^{(i j)}$ can be seen in [1].

Lemma 3.3. For $i, j=1, \ldots, m$ and $i \neq j$, the cross-covariance matrices of the innovations, $\Pi_{k}^{(i j)} \equiv E\left[\mu_{k}^{(i)} \mu_{k}^{(j) T}\right]$, are given by

$$
\begin{aligned}
\Pi_{k}^{(i j)}= & \Delta_{k}^{(i j)}+E\left[B_{k}^{(i)} \mathcal{E}_{k}^{(i) T} H_{k}^{(j) T}\right]+E\left[B_{k}^{(i)} R_{k}^{(i j)} B_{k}^{(j) T}\right] \\
& -\mathcal{V}_{k, k-1}^{(i j)} \Pi_{k-1}^{(j)-1}\left(\bar{H}_{k}^{(j)} \mathcal{X}_{k, k-1}^{(j)}+\mathcal{Y}_{k, k-1}^{(j)}\right)^{T} \\
& -\bar{H}_{k}^{(i)} L_{k}^{(i j)}-\mathcal{Y}_{k, k-1}^{(i)} \Pi_{k-1}^{(i)-1} \Pi_{k-1, k}^{(i j)}, \quad k \geq 2 \\
\Pi_{1}^{(i j)}= & \Delta_{1}^{(i j)}+E\left[B_{1}^{(i)} \mathcal{E}_{1}^{(i) T} H_{1}^{(j) T}\right]+E\left[B_{1}^{(i)} R_{1}^{(i j)} B_{1}^{(j) T}\right],
\end{aligned}
$$


where the expectations $\Delta_{k}^{(i j)} \equiv E\left[H_{k}^{(i)} x_{k} \mu_{k}^{(j) T}\right]$ are obtained by

$$
\begin{aligned}
\Delta_{k}^{(i j)}= & E\left[H_{k}^{(i)} \mathcal{D}_{k} H_{k}^{(j) T}\right]+E\left[H_{k}^{(i)} \mathcal{E}_{k}^{(j)} B_{k}^{(j) T}\right]-\bar{H}_{k}^{(i)} \bar{F}_{k-1} K_{k-1 / k-2}^{(j)} \bar{F}_{k-1}^{T} \bar{H}_{k}^{(j) T} \\
& -\Delta_{k, k-1}^{(i j)} \Pi_{k-1}^{(j)-1}\left(\bar{H}_{k}^{(j)} \mathcal{X}_{k, k-1}^{(j)}+\mathcal{Y}_{k, k-1}^{(j)}\right)^{T}, \quad k \geq 2 ; \\
\Delta_{1}^{(i j)}= & E\left[H_{1}^{(i)} \mathcal{D}_{1} H_{1}^{(j) T}\right]+E\left[H_{1}^{(i)} \mathcal{E}_{1}^{(j)} B_{1}^{(j) T}\right],
\end{aligned}
$$

and $\Pi_{k-1, k}^{(i j)} \equiv E\left[\mu_{k-1}^{(i)} \mu_{k}^{(j) T}\right]$ are given by

$$
\Pi_{k-1, k}^{(i j)}=\Delta_{k, k-1}^{(j i) T}+\mathcal{V}_{k, k-1}^{(j i) T}-L_{k, k-1}^{(j i) T} \bar{H}_{k}^{(j) T}-\Pi_{k-1}^{(i j)} \Pi_{k-1}^{(j)-1} \mathcal{Y}_{k, k-1}^{(j) T}, \quad k \geq 2 .
$$

Proof. As in Lemma 3.2, the measurement equation $y_{k}^{(i)}$ given in (1) and the innovation formula (6) will be repeatedly used.

Taking into account that $\Pi_{k}^{(i j)}=E\left[\mu_{k}^{(i)} \mu_{k}^{(j) T}\right]=E\left[y_{k}^{(i)} \mu_{k}^{(j) T}\right]-\bar{H}_{k}^{(i)} E\left[\widehat{x}_{k / k-1}^{(i)} \mu_{k}^{(j) T}\right]-$ $\mathcal{Y}_{k, k-1}^{(i)} \Pi_{k-1}^{(i)-1} E\left[\mu_{k-1}^{(i)} \mu_{k}^{(j) T}\right]$, it is easy to check that

$$
\Pi_{k}^{(i j)}=\Delta_{k}^{(i j)}+E\left[B_{k}^{(i)} v_{k}^{(i)} \mu_{k}^{(j) T}\right]-\bar{H}_{k}^{(i)} L_{k}^{(i j)}-\mathcal{Y}_{k, k-1}^{(i)} \Pi_{k-1}^{(i)-1} \Pi_{k, k-1}^{(j i) T} .
$$

To obtain $E\left[B_{k}^{(i)} v_{k}^{(i)} \mu_{k}^{(j) T}\right]$, we use (13) for the expectation $E\left[B_{k}^{(i)} v_{k}^{(i)} \widehat{x}_{k / k-1}^{(j) T}\right]$, which yields

$$
\begin{aligned}
E\left[B_{k}^{(i)} v_{k}^{(i)} \mu_{k}^{(j) T}\right]= & E\left[B_{k}^{(i)} \mathcal{E}_{k}^{(i) T} H_{k}^{(j) T}\right]+E\left[B_{k}^{(i)} R_{k}^{(i j)} B_{k}^{(j) T}\right] \\
& -\mathcal{V}_{k, k-1}^{(i j)} \Pi_{k-1}^{(j)-1} \mathcal{X}_{k, k-1}^{(j) T} \bar{H}_{k}^{(j) T}-\mathcal{V}_{k, k-1}^{(i j)} \Pi_{k-1}^{(j)-1} \mathcal{Y}_{k, k-1}^{(j) T}
\end{aligned}
$$

and expression (14) for $\Pi_{k}^{(i j)}$ is deduced.

To derive formula (15) for $\Delta_{k}^{(i j)}=E\left[H_{k}^{(i)} x_{k} \mu_{k}^{(j) T}\right]=E\left[H_{k}^{(i)} x_{k} y_{k}^{(j) T}\right]-$ $E\left[H_{k}^{(i)} x_{k} \widehat{x}_{k / k-1}^{(j) T}\right] \bar{H}_{k}^{(j) T}-E\left[H_{k}^{(i)} x_{k} \mu_{k-1}^{(j) T}\right] \Pi_{k-1}^{(j)-1} \mathcal{Y}_{k, k-1}^{(j) T}$, we use the conditional expectation properties to obtain

$\Delta_{k}^{(i j)}=E\left[H_{k}^{(i)} \mathcal{D}_{k} H_{k}^{(j) T}\right]+E\left[H_{k}^{(i)} \mathcal{E}_{k}^{(j)} B_{k}^{(j) T}\right]-E\left[H_{k}^{(i)} x_{k} \widehat{x}_{k / k-1}^{(j) T}\right] \bar{H}_{k}^{(j) T}-\Delta_{k, k-1}^{(i j)} \Pi_{k-1}^{(j)-1} \mathcal{Y}_{k, k-1}^{(j) T}$, and, using (12) for $E\left[H_{k}^{(i)} x_{k} \widehat{x}_{k / k-1}^{(j) T}\right]$, expression (15) is proved.

Finally, expression (16) for $\Pi_{k-1, k}^{(i j)}$ is obtained by using a similar reasoning to that used to derive $\Pi_{k}^{(i j)}$.

The distributed fusion filter, $\widehat{x}_{k / k}^{(D)}$, and its error covariance matrix, $\Sigma_{k / k}^{(D)}$, are given in the following theorem.

Theorem 3.4. Let $\widehat{X}_{k / k}=\left(\widehat{x}_{k / k}^{(1) T}, \ldots, \widehat{x}_{k / k}^{(m) T}\right)^{T}$ be the vector consisting of the local filters obtained in Theorem 3.1. Then, the distributed fusion filter for the system (1) 
is given by

$$
\widehat{x}_{k / k}^{(D)}=\Xi_{k / k} K_{k / k}^{-1} \widehat{X}_{k / k}, \quad k \geq 1
$$

where $K_{k / k}=\left(K_{k / k}^{(i j)}\right)_{i, j=1, \ldots, m}$ and $\Xi_{k / k}=\left(K_{k / k}^{(1)}, \ldots, K_{k / k}^{(m)}\right)$, with the crosscovariance matrices, $K_{k / k}^{(i j)}, i, j=1, \ldots, m$, between any two local filters calculated as

$$
\begin{aligned}
K_{k / k}^{(i j)}= & K_{k / k-1}^{(i j)}+L_{k}^{(i j)} \Pi_{k}^{(j)-1} \mathcal{X}_{k}^{(j) T}+\mathcal{X}_{k}^{(i)} \Pi_{k}^{(i)-1} L_{k}^{(j i) T} \\
& +\mathcal{X}_{k}^{(i)} \Pi_{k}^{(i)-1} \Pi_{k}^{(i j)} \Pi_{k}^{(j)-1} \mathcal{X}_{k}^{(j) T}, \quad k \geq 1, \quad i \neq j ; \\
K_{k / k}^{(i)}= & \mathcal{D}_{k}-\Sigma_{k / k}^{(i)}, \quad k \geq 1 .
\end{aligned}
$$

The error covariance matrices of the distributed fusion filtering estimators are computed by

$$
\Sigma_{k / k}^{(D)}=\mathcal{D}_{k}-\Xi_{k / k} K_{k / k}^{-1} \Xi_{k / k}^{T}, \quad k \geq 1
$$

Finally, the matrices $L_{k}^{(i j)}, K_{k / k-1}^{(i j)}$ and $\Pi_{k}^{(i j)}$ are given in Lemmas 3.2 and 3.3, respectively.

Proof. The proof can be seen in [1]. Hence the details are omitted here.

\section{Numerical simulation examples}

In this section we show that the results obtained in the current paper for the system model with random measurement matrices (1) can be applied to the multi-sensor systems with correlated randomly delayed measurements and missing measurements as particular cases.

\subsection{Multi-sensor system with randomly delayed measurements}

Consider a discrete-time linear stochastic system with state-dependent multiplicative noise and scalar randomly delayed measurements coming from two sensors:

$$
\begin{aligned}
& x_{k}=\left(0.95+0.2 \epsilon_{k-1}\right) x_{k-1}+w_{k-1}, \quad k \geq 1, \\
& z_{k}^{(i)}=C^{(i)} x_{k}+v_{k}^{(i)}, \quad k \geq 1, \quad i=1,2, \\
& y_{k}^{(i)}=\left(1-\gamma_{k}^{(i)}\right) z_{k}^{(i)}+\gamma_{k}^{(i)} z_{k-1}^{(i)}, \quad k \geq 2 ; \quad y_{1}^{(i)}=z_{1}^{(i)}, \quad i=1,2
\end{aligned}
$$

where $\left\{\epsilon_{k} ; k \geq 0\right\}$ is a zero-mean Gaussian white process with unit variance. Let us assume that $C^{(1)}=0.7$ and $C^{(2)}=0.6$. The additive noises are defined as $w_{k}=$ $0.6\left(\eta_{k}+\eta_{k+1}\right)$ and $v_{k}^{(i)}=c^{(i)} \eta_{k}, i=1,2$, where $c^{(1)}=1, c^{(2)}=0.5$ and $\left\{\eta_{k} ; k \geq 0\right\}$ is a zero-mean Gaussian white process with variance 0.5.

For $i=1,2$, the random variables $\gamma_{k}^{(i)}$, which model the random delays, are defined by two independent sequences of independent Bernoulli random variables, $\left\{\alpha_{k}^{(i)} ; k \geq\right.$ 
1\}, $i=1,2$, with probabilities $P\left[\alpha_{k}^{(i)}=1\right]=\bar{\alpha}^{(i)}$; specifically, for $i=1,2, \gamma_{k}^{(i)}=$ $\alpha_{k+1}^{(i)}\left(1-\alpha_{k}^{(i)}\right)$. Due to this definition, if $\gamma_{k}^{(i)}=1$, then $\gamma_{k+1}^{(i)}=0$. Therefore, two consecutive delays cannot happen in the transmission of the data.

Taking into account the previous definition, at each sensor the variables $\gamma_{k}^{(i)}$ and $\gamma_{s}^{(i)}$ are independent for $|k-s| \neq 0,1$ but correlated at consecutive sampling times. The mean of these variables is $\bar{\gamma}^{(i)}=\bar{\alpha}^{(i)}\left(1-\bar{\alpha}^{(i)}\right), i=1,2$, and the correlation function is given by $E\left[\gamma_{k}^{(i)} \gamma_{s}^{(i)}\right]=\left\{\begin{array}{ll}\bar{\gamma}^{(i)}, & |k-s|=0 \\ 0, & |k-s|=1\end{array}\right.$.

In order to apply the theoretical results established in Section 3, system (17) can be equivalently rewritten as the following one, with random parameter matrices:

$$
\begin{aligned}
& X_{k+1}=\mathcal{F}_{k} X_{k}+W_{k}, \quad k \geq 0, \\
& y_{k}^{(i)}=H_{k}^{(i)} X_{k}+B_{k}^{(i)} V_{k}^{(i)}, \quad k \geq 1, \quad i=1,2,
\end{aligned}
$$

where

$$
\begin{aligned}
& X_{k}=\left(\begin{array}{c}
x_{k} \\
x_{k-1}
\end{array}\right), \quad k \geq 1 ; \quad X_{0}=\left(\begin{array}{c}
x_{0} \\
0
\end{array}\right) . \\
& \mathcal{F}_{k}=\left(\begin{array}{cc}
0.95+0.2 \epsilon_{k} & 0 \\
1 & 0
\end{array}\right), \quad W_{k}=\left(\begin{array}{c}
w_{k} \\
0
\end{array}\right), \quad k \geq 0 . \\
& H_{k}^{(i)}=\left\{\begin{array}{cc}
\left(C^{(i)}, 0\right), & k=1, \\
\left(\left(1-\gamma_{k}^{(i)}\right) C^{(i)}, \gamma_{k}^{(i)} C^{(i)}\right), & k \geq 2,
\end{array} B_{k}^{(i)}=\left\{\begin{array}{cc}
(1,0), & k=1, \\
\left(1-\gamma_{k}^{(i)}, \gamma_{k}^{(i)}\right), & k \geq 2 .
\end{array}\right.\right. \\
& V_{k}^{(i)}=\left\{\begin{array}{cl}
\left(v_{1}^{(i)}, 0\right)^{T}, & k=1, \\
\left(v_{k}^{(i)}, v_{k-1}^{(i)}\right)^{T}, & k \geq 2 .
\end{array}\right.
\end{aligned}
$$

The new noise processes and the random parameter matrices of system (18) satisfy the assumptions 1-4 to apply the algorithm proposed in this paper. Specifically, we have:

- $\left\{H_{k}^{(i)} ; k \geq 1\right\}$ and $\left\{B_{k}^{(i)} ; k \geq 1\right\}$ are correlated and cross-correlated at consecutive sampling times. 
- The additive noises $\left\{W_{k} ; k \geq 0\right\}$ and $\left\{V_{k}^{(i)} ; k \geq 1\right\}$ are correlated, with

$$
\begin{aligned}
& \circ Q_{k}=\left(\begin{array}{cc}
0.36 & 0 \\
0 & 0
\end{array}\right), k \geq 0, \quad Q_{k, k-1}=\left(\begin{array}{cc}
0.18 & 0 \\
0 & 0
\end{array}\right), k \geq 1 . \\
& \circ R_{k}^{(i j)}=\left(\begin{array}{cc}
0.5 c^{(i)} c^{(j)} & 0 \\
0 & 0.5 c^{(i)} c^{(j)}
\end{array}\right), k \geq 2 ; \quad R_{1}^{(i j)}=\left(\begin{array}{cc}
0.5 c^{(i)} c^{(j)} & 0 \\
0 & 0
\end{array}\right), \\
& R_{k, k-1}^{(i j)}=\left(\begin{array}{cc}
0 & 0 \\
0.5 c^{(i)} c^{(j)} & 0
\end{array}\right), k \geq 2 . \\
& \circ S_{k}^{(i)}=\left(\begin{array}{cc}
0.3 c^{(i)} & 0 \\
0 & 0
\end{array}\right), k \geq 1, \\
& S_{k-1, k}^{(i)}=\left(\begin{array}{cc}
0.3 c^{(i)} & 0.3 c^{(i)} \\
0 & 0
\end{array}\right), k \geq 2 ; \quad S_{0,1}^{(i)}=S_{1}^{(i)}, \\
& S_{k-2, k}^{(i)}=\left(\begin{array}{cc}
0 & 0.3 c^{(i)} \\
0 & 0
\end{array}\right), k \geq 2 .
\end{aligned}
$$

To illustrate the feasibility of the proposed estimators, the corresponding algorithms were implemented in MATLAB, and one hundred iterations of the centralized linear filtering algorithm [12] and the proposed distributed filtering algorithm were run. The error variances of both distributed and centralized fusion filters were calculated for several values of $\bar{\alpha}^{(i)}$ which provide different values of the delay probabilities $\bar{\gamma}^{(i)}, i=$ 1,2 . Only the cases $\bar{\alpha}^{(i)} \leq 0.5$ are displayed due to the symmetry of $\bar{\gamma}^{(i)}=\bar{\alpha}^{(i)}\left(1-\bar{\alpha}^{(i)}\right)$.

Figure 1 shows local, centralized, and distributed filtering error variances considering $\bar{\alpha}^{(1)}=0.5$ and $\bar{\alpha}^{(2)}=0.1$. This figure confirms that the distributed fusion filter outperforms each local filter and that the centralized method is more accurate than the distributed one.

Next, we will show the dependence of the estimation error variances upon the values $\bar{\alpha}^{(1)}$ and $\bar{\alpha}^{(2)}$. Since in all the cases examined, the error variances present insignificant variation from a certain iteration onwards, Figure 2 displays the filtering error variances, at a fixed iteration (namely, $k=100$ ), when both $\bar{\alpha}^{(1)}$ and $\bar{\alpha}^{(2)}$ are varied from 0.1 to 0.5 , which provide different values of the delay probabilities $\bar{\gamma}^{(1)}$ and $\bar{\gamma}^{(2)}$ $\left(\bar{\gamma}^{(i)}=0.09,0.16,0.21,0.24,0.25\right)$. In both graphs we can see that worse estimations are obtained as the delay probabilities increase. Also, this figure confirms the similar accuracy of both methods, centralized and distributed.

\subsection{Multi-sensor system with missing measurements}

Let $\left\{x_{k} ; k \geq 0\right\}$ be the state process generated by the same model as that in Section 4.1 and let us consider missing measurements coming from two sensors given by

$$
y_{k}^{(i)}=\theta_{k}^{(i)} C^{(i)} x_{k}+v_{k}^{(i)}, \quad k \geq 1, \quad i=1,2
$$




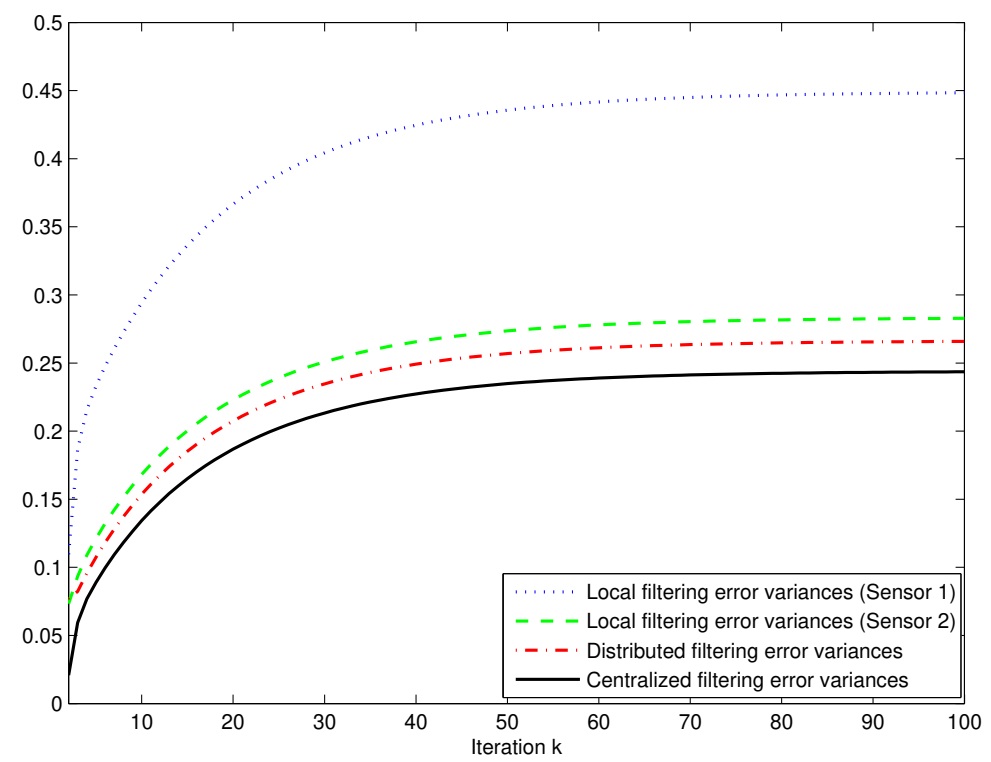

Figure 1. Filtering error variances for $\bar{\alpha}^{(1)}=0.5$ and $\bar{\alpha}^{(2)}=0.1$.
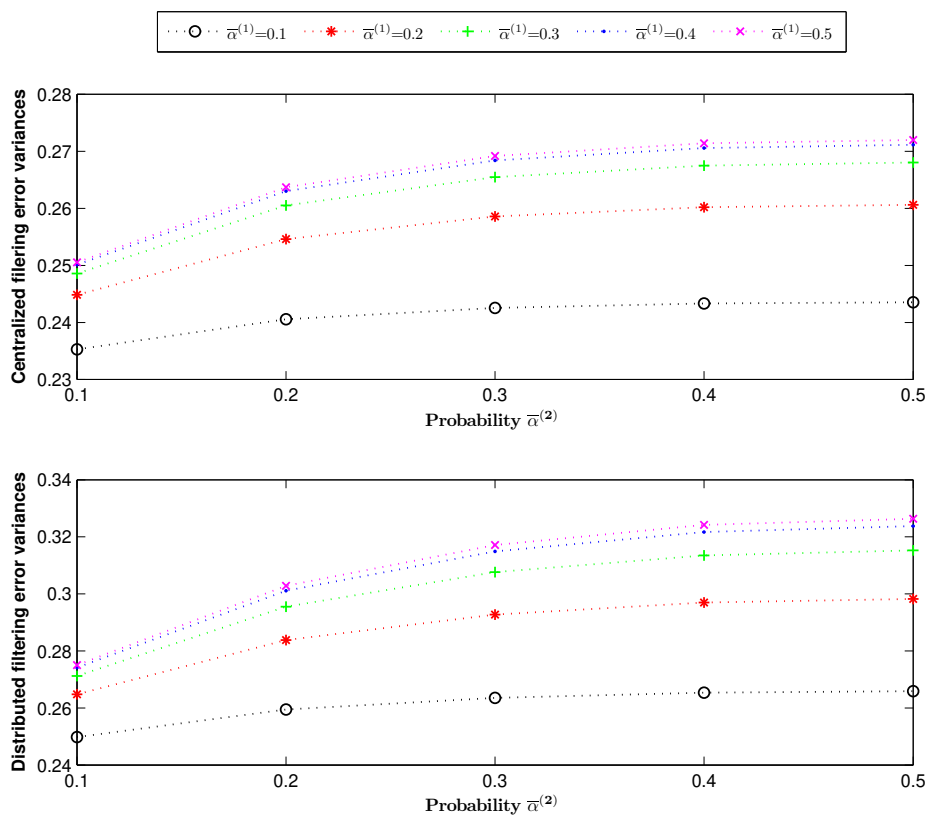

Figure 2. Centralized and distributed filtering error variances at $k=100$ versus $\bar{\alpha}^{(2)}$ with $\bar{\alpha}^{(1)}$ varying from 0.1 to 0.5 .

The additive noises are defined by $v_{k}^{(i)}=c^{(i)}\left(\eta_{k-1}+\eta_{k}\right), i=1,2$, where $c^{(1)}=0.75$ and $c^{(2)}=0.5$. It is assumed that $C^{(1)}, C^{(2)}$ and the process $\left\{\eta_{k} ; k \geq 1\right\}$ are defined in Section 4.1.

The random variables $\left\{\theta_{k}^{(1)} ; k \geq 1\right\}$ used to model the missing phenomenon in the first sensor are defined by a sequence of independent Bernoulli random variables, 
$\left\{\beta_{k} ; k \geq 0\right\}$, with probabilities $P\left[\beta_{k}=1\right]=\bar{\beta}$; specifically, $\theta_{k}^{(1)}=1-\beta_{k-1}\left(1-\beta_{k}\right)$. Taking into account this relation, the variables $\theta_{k}^{(1)}$ and $\theta_{s}^{(1)}$ are independent for $\mid k-$ $s \mid \geq 2$. Also, it is easy to check that if $\theta_{k}^{(1)}=0$, then $\theta_{k+1}^{(1)}=1$, hence, in this sensor the state cannot be missing in two successive observations. The mean of these variables is given by $\bar{\theta}=1-\bar{\beta}(1-\bar{\beta})$ and the correlation function is

$$
E\left[\theta_{k}^{(1)} \theta_{s}^{(1)}\right]= \begin{cases}\bar{\theta}, & |k-s|=0 \\ 2 \bar{\theta}-1, & |k-s|=1\end{cases}
$$

In the second sensor, the missing phenomenon is modelled by a sequence of independent random variables $\left\{\theta_{k}^{(2)} ; k \geq 1\right\}$ with probability distribution $P\left[\theta_{k}^{(2)}=0\right]=0.1$, $P\left[\theta_{k}^{(2)}=0.5\right]=0.5$ and $P\left[\theta_{k}^{(2)}=1\right]=0.4$.

Under these assumptions, it can be easily seen that the measurement equations (19) are a particular case of those in (1) with $H_{k}^{(i)}=\theta_{k}^{(i)} C^{(i)}$ and $B_{k}^{(i)}=1$, for $k \geq 1$.

The additive noises $\left\{v_{k}^{(i)} ; k \geq 1\right\}$ are one-step autocorrelated between the different sensors and two-step cross-correlated with $\left\{w_{k} ; k \geq 0\right\}$ and

$$
\begin{aligned}
& R_{k}^{(i j)}=c^{(i)} c^{(j)}, \quad k \geq 1 ; \quad R_{k, k-1}^{(i j)}=0.5 c^{(i)} c^{(j)}, \quad k \geq 2 . \\
& S_{k}^{(i)}=0.3 c^{(i)}, \quad S_{k-1, k}^{(i)}=0.6 c^{(i)}, \quad k \geq 1 ; \quad S_{k-2 . k}^{(i)}=0.3 c^{(i)}, \quad k \geq 2 .
\end{aligned}
$$

The accuracy of the proposed local and distributed estimators in comparison with the centralized ones in [12] is studied. Figure 3 displays the error variances of the local, centralized and distributed fusion filters at a specific iteration $(k=100)$, for different values of $\bar{\beta}$ which provide different values of $\bar{\theta}$. Since the value of $\bar{\theta}$ does not vary if the value $1-\bar{\beta}$ is considered instead of $\bar{\beta}$, it is enough to consider $\bar{\beta} \leq 0.5$. This figure corroborates that the distributed filter performs quite better than the local filters, but lightly worse than the centralized filter; however, the performance of both filters is similar and provide good estimations. Let us observe that, as the probability $\bar{\beta}$ increases (which means that the probability $1-\bar{\theta}$ of only-noise measurements increases in the first sensor), the filtering error variances become greater and, consequently, worse estimations are obtained. Note that the local filtering error variances at the second sensor are constant, because its behaviour is not influenced by $\bar{\beta}$.

\section{Conclusion}

The distributed fusion filtering problem has been addressed for multi-sensor stochastic systems with correlated random parameter matrices and additive noises. Firstly, recursive algorithms for the local LS linear filters of the system state based on the measurements coming from each sensor have been obtained. Next, a distributed fusion filter has been designed as a matrix-weighted linear combination of such local estimators by minimizing the mean squared estimation error. The applicability of the proposed estimators has been illustrated by two numerical simulation examples, where an error variance comparison has been carried out to show the performance of the centralized and distributed fusion estimators. 


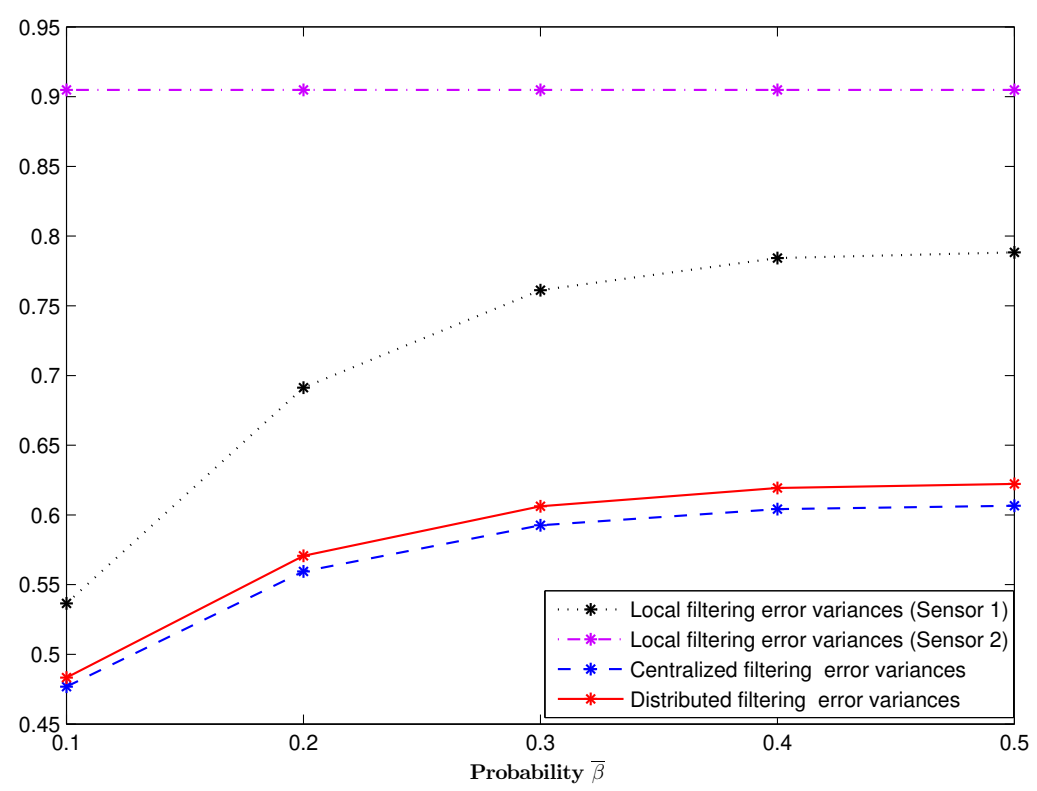

Figure 3. Local, centralized and distributed filtering error variances at $k=100$ versus $\bar{\beta}$.

\section{Disclosure statement}

No potential conflict of interest was reported by the authors.

\section{Funding}

This work is supported by Ministerio de Economía y Competitividad and Fondo Europeo de Desarrollo Regional FEDER (grant no. MTM2014-52291-P and MTM201784199-P).

\section{ORCID}

R. Caballero-Águila http://orcid.org/0000-0001-7659-7649

I. García-Garrido http://orcid.org/0000-0003-3101-4088

J. Linares-Pérez http://orcid.org/0000-0002-6853-555X

\section{References}

[1] R. Caballero-Águila, I. García-Garrido and J. Linares-Pérez, Distributed fusion filtering in networked systems with random measurement matrices and correlated noises, Discret. Dyn. Nat. Soc. 2015 (2015) Article ID 398605, 10 pages.

[2] R. Caballero-Águila, A. Hermoso-Carazo and J. Linares-Pérez, Networked fusion filtering from outputs with stochastic uncertainties and correlated random transmission delays, Sensors 16 (2016), pp. 1-18. 
[3] R. Caballero-Águila, A. Hermoso-Carazo and J. Linares-Pérez, Fusion estimation using measured outputs with random parameter matrices subject to random delays and packet dropouts, Signal Process. 127 (2016), pp. 12-23.

[4] R. Caballero-Águila, A. Hermoso-Carazo and J. Linares-Pérez, New distributed fusion filtering algorithm based on covariances over sensor networks with random packet dropouts, Int. J. Syst. Sci. 48 (2017), pp. 1805-1817.

[5] R. Caballero-Águila, A. Hermoso-Carazo and J. Linares-Pérez, Covariance-based fusion filtering for networked systems with random transmission delays and non-consecutive losses, Int. J. Gen. Syst. 46 (2017), pp. 752-771.

[6] D. Chen, Y. Yu, L. Xu and X. Liu, Kalman filtering for discrete stochastic systems with multiplicative noises and random two-step sensor delays, Discret. Dyn. Nat. Soc. 2015 (2015) Article ID 809734, 11 pages.

[7] D. Chen, L. Xu and J. Du, Optimal filtering for systems with finite-step autocorrelated process noises, random one-step sensor delay and missing measurements, Commun. Nonlinear Sci. Numer. Simul. 32 (2016), pp. 211-224.

[8] J. Hu, Z. Wang and H. Gao, Recursive filtering with random parameter matrices, multiple fading measurements and correlated noises, Automatica 49 (2013), pp. 3440-3448.

[9] J. Hu, Z. Wang, S. Liu and H. Gao, A variance-constrained approach to recursive state estimation for time-varying complex networks with missing measurements, Automatica 64 (2016), pp. 155-162.

[10] J. Hu, Z. Wang. B. Shen and H. Gao, Quantised recursive filtering for a class of nonlinear systems with multiplicative noises and missing measurements, International Journal of Control 86 (2013), pp. 650-663.

[11] W. Li, Z. Wang, G. Wei, L. Ma, J. Hu and D. Ding, A Survey on multisensor fusion and consensus filtering for sensor networks, Discret. Dyn. Nat. Soc. 2015 (2015) Article ID 683701, 12 pages.

[12] J. Linares-Pérez, R. Caballero-Águila and I. García-Garrido, Optimal linear filter design for systems with correlation in the measurement matrices and noises: recursive algorithm and applications, Int. J. Syst. Sci. 45 (2014), pp. 1548-1562.

[13] Y. Luo, Y. Zhu, D. Luo, J. Zhou, E. Song and D. Wang, Globally optimal multisensor distributed random parameter matrices Kalman filtering fusion with applications, Sensors 8 (2008), pp. 8086-8103.

[14] J. Ma and S. Sun, Information fusion estimators for systems with multiple sensors of different packet dropout rates, Inf. Fusion 12 (2011), pp. 213-222.

[15] J. Ma and S. Sun, Distributed fusion filter for networked stochastic uncertain systems with transmission delays and packet dropouts, Signal Process. 130 (2017), pp. 268-278.

[16] F. Peng and S. Sun, Distributed fusion estimation for multisensor multirate systems with stochastic observation multiplicative noises, Math. Probl. Eng. 2014 (2014) Article ID 373270, 8 pages.

[17] S. Sun, H. Lin, J. Ma and X. Li, Multi-sensor distributed fusion estimation with applications in networked systems: A review paper, Inf. Fusion 38 (2017), pp. 122-134.

[18] S. Sun, T. Tian and L. Honglei, State estimators for systems with random parameter matrices, stochastic nonlinearities, fading measurements and correlated noises, Inf. Sci. 397-398 (2017), pp. 118-136.

[19] T. Tian, S. Sun and N. Li, Multi-sensor information fusion estimators for stochastic uncertain systems with correlated noises, Inf. Fusion 27 (2016), pp. 126-137.

[20] S. Wang, H. Fang and X. Liu, Distributed state estimation for stochastic non-linear systems with random delays and packet dropouts, IET Control Theory Appl. 9 (2015), pp. $2657-2665$.

[21] S. Wang, H. Fang and X. Tian, Recursive estimation for nonlinear stochastic systems with multi-step transmission delays, multiple packet dropouts and correlated noises, Signal Process. 115 (2015), pp. 164-175. 